\title{
Probability on Finite Set and Real-Valued Random Variables
}

\author{
Hiroyuki Okazaki \\ Shinshu University \\ Nagano, Japan
}

\author{
Yasunari Shidama \\ Shinshu University \\ Nagano, Japan
}

\begin{abstract}
Summary. In the various branches of science, probability and randomness provide us with useful theoretical frameworks. The Formalized Mathematics has already published some articles concerning the probability: [23], [24], [25], and [30]. In order to apply those articles, we shall give some theorems concerning the probability and the real-valued random variables to prepare for further studies.
\end{abstract}

MML identifier: RANDOM_1, version: $\underline{7.11 .02 \quad 4.120 .1050}$

The articles [12], [28], [3], [14], [1], [18], [27], [9], [29], [11], [4], [21], [10], [2], [5], [6], [20], [25], [24], [30], [7], [16], [17], [19], [8], [15], [26], [13], and [22] provide the notation and terminology for this paper.

\section{Probability on Finite Set}

One can prove the following four propositions:

(1) Let $X$ be a non empty set, $S_{1}$ be a $\sigma$-field of subsets of $X, M$ be a $\sigma$-measure on $S_{1}, f$ be a partial function from $X$ to $\overline{\mathbb{R}}, E$ be an element of $S_{1}$, and $a$ be a real number. Suppose $f$ is integrable on $M$ and $E \subseteq \operatorname{dom} f$ and $M(E)<+\infty$ and for every element $x$ of $X$ such that $x \in E$ holds $a \leq f(x)$. Then $\overline{\mathbb{R}}(a) \cdot M(E) \leq \int f\lceil E \mathrm{~d} M$.

(2) Let $X$ be a non empty set, $S_{1}$ be a $\sigma$-field of subsets of $X, M$ be a $\sigma$-measure on $S_{1}, f$ be a partial function from $X$ to $\mathbb{R}, E$ be an element of $S_{1}$, and $a$ be a real number. Suppose $f$ is integrable on $M$ and $E \subseteq \operatorname{dom} f$ and $M(E)<+\infty$ and for every element $x$ of $X$ such that $x \in E$ holds $a \leq f(x)$. Then $\overline{\mathbb{R}}(a) \cdot M(E) \leq \int f\lceil E \mathrm{~d} M$. 
(3) Let $X$ be a non empty set, $S_{1}$ be a $\sigma$-field of subsets of $X, M$ be a $\sigma$-measure on $S_{1}, f$ be a partial function from $X$ to $\overline{\mathbb{R}}, E$ be an element of $S_{1}$, and $a$ be a real number. Suppose $f$ is integrable on $M$ and $E \subseteq \operatorname{dom} f$ and $M(E)<+\infty$ and for every element $x$ of $X$ such that $x \in E$ holds $f(x) \leq a$. Then $\int f\lceil E \mathrm{~d} M \leq \overline{\mathbb{R}}(a) \cdot M(E)$.

(4) Let $X$ be a non empty set, $S_{1}$ be a $\sigma$-field of subsets of $X, M$ be a $\sigma$-measure on $S_{1}, f$ be a partial function from $X$ to $\mathbb{R}, E$ be an element of $S_{1}$, and $a$ be a real number. Suppose $f$ is integrable on $M$ and $E \subseteq \operatorname{dom} f$ and $M(E)<+\infty$ and for every element $x$ of $X$ such that $x \in E$ holds $f(x) \leq a$. Then $\int f\lceil E \mathrm{~d} M \leq \overline{\mathbb{R}}(a) \cdot M(E)$.

\section{RANDOM VARIABLES}

For simplicity, we follow the rules: $O$ is a non empty set, $r$ is a real number, $S$ is a $\sigma$-field of subsets of $O, P$ is a probability on $S$, and $E$ is a finite non empty set.

Let $E$ be a non empty set. We introduce the trivial $\sigma$-field of $E$ as a synonym of $2^{E}$. Then the trivial $\sigma$-field of $E$ is a $\sigma$-field of subsets of $E$.

Next we state a number of propositions:

(5) Let $O$ be a non empty finite set and $f$ be a partial function from $O$ to $\mathbb{R}$. Then there exists a finite sequence $F$ of separated subsets of the trivial $\sigma$-field of $O$ and there exists a finite sequence $s$ of elements of $\operatorname{dom} f$ such that

$\operatorname{dom} f=\bigcup \operatorname{rng} F$ and $\operatorname{dom} F=\operatorname{dom} s$ and $s$ is one-to-one and $\operatorname{rng} s=$ $\operatorname{dom} f$ and len $s=\overline{\overline{\operatorname{dom} f}}$ and for every natural number $k$ such that $k \in$ $\operatorname{dom} F$ holds $F(k)=\{s(k)\}$ and for every natural number $n$ and for all elements $x, y$ of $O$ such that $n \in \operatorname{dom} F$ and $x, y \in F(n)$ holds $f(x)=f(y)$.

(6) Let $O$ be a non empty finite set and $f$ be a partial function from $O$ to $\mathbb{R}$. Then

(i) $\quad f$ is simple function in the trivial $\sigma$-field of $O$, and

(ii) $\operatorname{dom} f$ is an element of the trivial $\sigma$-field of $O$.

(7) Let $O$ be a non empty finite set, $M$ be a $\sigma$-measure on the trivial $\sigma$ field of $O$, and $f$ be a partial function from $O$ to $\mathbb{R}$. If $\operatorname{dom} f \neq \emptyset$ and $M(\operatorname{dom} f)<+\infty$, then $f$ is integrable on $M$.

(8) Let $O$ be a non empty finite set and $f$ be a partial function from $O$ to $\mathbb{R}$. Then there exists an element $X$ of the trivial $\sigma$-field of $O$ such that $\operatorname{dom} f=X$ and $f$ is measurable on $X$.

(9) Let $O$ be a non empty finite set, $M$ be a $\sigma$-measure on the trivial $\sigma$-field of $O, f$ be a function from $O$ into $\mathbb{R}, x$ be a finite sequence of elements of $\overline{\mathbb{R}}$, and $s$ be a finite sequence of elements of $O$. Suppose $M(O)<+\infty$ and 
$s$ is one-to-one and $\operatorname{rng} s=O$ and len $s=\overline{\bar{O}}$. Then there exists a finite sequence $F$ of separated subsets of the trivial $\sigma$-field of $O$ and there exists a finite sequence $a$ of elements of $\mathbb{R}$ such that

(i) $\operatorname{dom} f=\bigcup \operatorname{rng} F$,

(ii) $\operatorname{dom} a=\operatorname{dom} s$,

(iii) $\operatorname{dom} F=\operatorname{dom} s$,

(iv) for every natural number $k$ such that $k \in \operatorname{dom} F$ holds $F(k)=\{s(k)\}$, and

(v) for every natural number $n$ and for all elements $x, y$ of $O$ such that $n \in \operatorname{dom} F$ and $x, y \in F(n)$ holds $f(x)=f(y)$.

(10) Let $O$ be a non empty finite set, $M$ be a $\sigma$-measure on the trivial $\sigma$-field of $O, f$ be a function from $O$ into $\mathbb{R}, x$ be a finite sequence of elements of $\overline{\mathbb{R}}$, and $s$ be a finite sequence of elements of $O$. Suppose that

(i) $M(O)<+\infty$

(ii) $\operatorname{len} x=\overline{\bar{O}}$,

(iii) $s$ is one-to-one,

(iv) $\operatorname{rng} s=O$,

(v) $\quad$ len $s=\overline{\bar{O}}$, and

(vi) for every natural number $n$ such that $n \in \operatorname{dom} x$ holds $x(n)=$ $\overline{\mathbb{R}}(f(s(n))) \cdot M(\{s(n)\})$.

Then $\int f \mathrm{~d} M=\sum x$.

(11) Let $O$ be a non empty finite set, $M$ be a $\sigma$-measure on the trivial $\sigma$-field of $O$, and $f$ be a function from $O$ into $\mathbb{R}$. Suppose $M(O)<+\infty$. Then there exists a finite sequence $x$ of elements of $\overline{\mathbb{R}}$ and there exists a finite sequence $s$ of elements of $O$ such that

(i) $\operatorname{len} x=\overline{\bar{O}}$,

(ii) $s$ is one-to-one,

(iii) $\operatorname{rng} s=O$,

(iv) $\operatorname{len} s=\overline{\bar{O}}$,

(v) for every natural number $n$ such that $n \in \operatorname{dom} x$ holds $x(n)=$ $\overline{\mathbb{R}}(f(s(n))) \cdot M(\{s(n)\})$, and

(vi) $\int f \mathrm{~d} M=\sum x$.

(12) Let $O$ be a non empty finite set, $P$ be a probability on the trivial $\sigma$-field of $O, f$ be a function from $O$ into $\mathbb{R}, x$ be a finite sequence of elements of $\mathbb{R}$, and $s$ be a finite sequence of elements of $O$. Suppose that

(i) $\operatorname{len} x=\overline{\bar{O}}$,

(ii) $s$ is one-to-one,

(iii) $\operatorname{rng} s=O$,

(iv) len $s=\overline{\bar{O}}$, and

(v) for every natural number $n$ such that $n \in \operatorname{dom} x$ holds $x(n)=f(s(n))$. $P(\{s(n)\})$. 
Then $\int f \mathrm{~d} \mathrm{P} 2 \mathrm{M} P=\sum x$.

(13) Let $O$ be a non empty finite set, $P$ be a probability on the trivial $\sigma$-field of $O$, and $f$ be a function from $O$ into $\mathbb{R}$. Then there exists a finite sequence $F$ of elements of $\mathbb{R}$ and there exists a finite sequence $s$ of elements of $O$ such that

(i) $\operatorname{len} F=\overline{\bar{O}}$,

(ii) $s$ is one-to-one,

(iii) $\operatorname{rng} s=O$,

(iv) $\quad$ len $s=\overline{\bar{O}}$,

(v) for every natural number $n$ such that $n \in \operatorname{dom} F$ holds $F(n)=f(s(n))$. $P(\{s(n)\})$, and

(vi) $\int f \mathrm{~d} \mathrm{P} 2 \mathrm{MP}=\sum F$.

(14) Let $E$ be a finite non empty set and $A$ be a sequence of subsets of $E$. Suppose $A$ is non-increasing. Then there exists an element $N$ of $\mathbb{N}$ such that for every element $m$ of $\mathbb{N}$ such that $N \leq m$ holds $A(N)=A(m)$.

(15) Let $E$ be a finite non empty set and $A$ be a sequence of subsets of $E$. Suppose $A$ is non-increasing. Then there exists an element $N$ of $\mathbb{N}$ such that for every element $m$ of $\mathbb{N}$ such that $N \leq m$ holds Intersection $A=A(m)$.

(16) Let $E$ be a finite non empty set and $A$ be a sequence of subsets of $E$. Suppose $A$ is non-decreasing. Then there exists an element $N$ of $\mathbb{N}$ such that for every element $m$ of $\mathbb{N}$ such that $N \leq m$ holds $A(N)=A(m)$.

(17) Let $E$ be a finite non empty set and $A$ be a sequence of subsets of $E$. Suppose $A$ is non-decreasing. Then there exists a natural number $N$ such that for every natural number $m$ such that $N \leq m$ holds $\bigcup A=A(m)$.

Let us consider $E$. The trivial probability of $E$ yielding a probability on the trivial $\sigma$-field of $E$ is defined as follows:

(Def. 1) For every event $A_{1}$ of $E$ holds (the trivial probability of $\left.E\right)\left(A_{1}\right)=\mathrm{P}\left(A_{1}\right)$.

Let us consider $O, S$. A function from $O$ into $\mathbb{R}$ is said to be a real-valued random variable of $S$ if:

(Def. 2) There exists an element $X$ of $S$ such that $X=O$ and it is measurable on $X$.

In the sequel $f, g$ are real-valued random variables of $S$.

Next we state the proposition

(18) $f+g$ is a real-valued random variable of $S$.

Let us consider $O, S, f, g$. Then $f+g$ is a real-valued random variable of $S$.

We now state the proposition

(19) $f-g$ is a real-valued random variable of $S$.

Let us consider $O, S, f, g$. Then $f-g$ is a real-valued random variable of $S$.

Next we state the proposition

(20) For every real number $r$ holds $r f$ is a real-valued random variable of $S$. 
Let us consider $O, S, f$ and let $r$ be a real number. Then $r f$ is a real-valued random variable of $S$.

Next we state two propositions:

(21) For all partial functions $f, g$ from $O$ to $\mathbb{R}$ holds $\overline{\mathbb{R}}(f) \overline{\mathbb{R}}(g)=\overline{\mathbb{R}}(f g)$.

(22) $f g$ is a real-valued random variable of $S$.

Let us consider $O, S, f, g$. Then $f g$ is a real-valued random variable of $S$.

Next we state two propositions:

(23) For every real number $r$ such that $0 \leq r$ and $f$ is non-negative holds $f^{r}$ is a real-valued random variable of $S$.

(24) $|f|$ is a real-valued random variable of $S$.

Let us consider $O, S, f$. Then $|f|$ is a real-valued random variable of $S$.

We now state the proposition

(25) For every real number $r$ such that $0 \leq r$ holds $|f|^{r}$ is a real-valued random variable of $S$.

Let us consider $O, S, f, P$. We say that $f$ is integrable on $P$ if and only if:

(Def. 3) $f$ is integrable on $\mathrm{P} 2 \mathrm{M} P$.

Let us consider $O, S, P$ and let $f$ be a real-valued random variable of $S$. Let us assume that $f$ is integrable on $P$. The functor $E_{P}\{f\}$ yielding an element of $\mathbb{R}$ is defined as follows:

(Def. 4) $E_{P}\{f\}=\int f \mathrm{~d} \mathrm{P} 2 \mathrm{M} P$.

One can prove the following propositions:

(26) If $f$ is integrable on $P$ and $g$ is integrable on $P$, then $E_{P}\{f+g\}=$ $E_{P}\{f\}+E_{P}\{g\}$.

(27) If $f$ is integrable on $P$, then $E_{P}\{r f\}=r \cdot E_{P}\{f\}$.

(28) If $f$ is integrable on $P$ and $g$ is integrable on $P$, then $E_{P}\{f-g\}=$ $E_{P}\{f\}-E_{P}\{g\}$.

(29) For every non empty finite set $O$ holds every function from $O$ into $\mathbb{R}$ is a real-valued random variable of the trivial $\sigma$-field of $O$.

(30) Let $O$ be a non empty finite set, $P$ be a probability on the trivial $\sigma$-field of $O$, and $X$ be a real-valued random variable of the trivial $\sigma$-field of $O$. Then $X$ is integrable on $P$.

(31) Let $O$ be a non empty finite set, $P$ be a probability on the trivial $\sigma$-field of $O, X$ be a real-valued random variable of the trivial $\sigma$-field of $O, F$ be a finite sequence of elements of $\mathbb{R}$, and $s$ be a finite sequence of elements of $O$. Suppose that

(i) $\operatorname{len} F=\overline{\bar{O}}$,

(ii) $s$ is one-to-one,

(iii) $\operatorname{rng} s=O$,

(iv) len $s=\overline{\bar{O}}$, and 
(v) for every natural number $n$ such that $n \in \operatorname{dom} F$ holds $F(n)=X(s(n))$. $P(\{s(n)\})$.

Then $E_{P}\{X\}=\sum F$.

(32) Let $O$ be a non empty finite set, $P$ be a probability on the trivial $\sigma$-field of $O$, and $X$ be a real-valued random variable of the trivial $\sigma$-field of $O$. Then there exists a finite sequence $F$ of elements of $\mathbb{R}$ and there exists a finite sequence $s$ of elements of $O$ such that

(i) $\operatorname{len} F=\overline{\bar{O}}$,

(ii) $s$ is one-to-one,

(iii) $\operatorname{rng} s=O$,

(iv) $\quad$ len $s=\overline{\bar{O}}$,

(v) for every natural number $n$ such that $n \in \operatorname{dom} F$ holds $F(n)=X(s(n))$. $P(\{s(n)\})$, and

(vi) $\quad E_{P}\{X\}=\sum F$.

(33) Let $O$ be a non empty finite set, $P$ be a probability on the trivial $\sigma$-field of $O$, and $X$ be a real-valued random variable of the trivial $\sigma$-field of $O$. Then there exists a finite sequence $F$ of elements of $\mathbb{R}$ and there exists a finite sequence $s$ of elements of $O$ such that

(i) $\operatorname{len} F=\overline{\bar{O}}$,

(ii) $s$ is one-to-one,

(iii) $\operatorname{rng} s=O$,

(iv) len $s=\overline{\bar{O}}$,

(v) for every natural number $n$ such that $n \in \operatorname{dom} F$ holds $F(n)=X(s(n))$. $P(\{s(n)\})$, and

(vi) $E_{P}\{X\}=\sum F$.

(34) Let $O$ be a non empty finite set, $X$ be a real-valued random variable of the trivial $\sigma$-field of $O, G$ be a finite sequence of elements of $\mathbb{R}$, and $s$ be a finite sequence of elements of $O$. Suppose len $G=\overline{\bar{O}}$ and $s$ is one-to-one and $\operatorname{rng} s=O$ and len $s=\overline{\bar{O}}$ and for every natural number $n$ such that

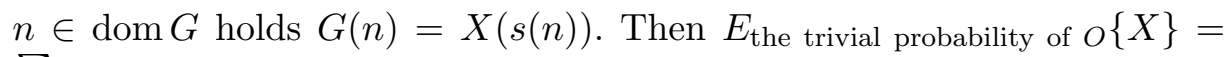
$\frac{\sum G}{\overline{\bar{O}}}$.

(35) Let $O$ be a non empty finite set and $X$ be a real-valued random variable of the trivial $\sigma$-field of $O$. Then there exists a finite sequence $G$ of elements of $\mathbb{R}$ and there exists a finite sequence $s$ of elements of $O$ such that

(i) $\operatorname{len} G=\overline{\bar{O}}$,

(ii) $s$ is one-to-one,

(iii) $\operatorname{rng} s=O$,

(iv) $\quad$ len $s=\overline{\bar{O}}$,

(v) for every natural number $n$ such that $n \in \operatorname{dom} G$ holds $G(n)=X(s(n))$, and 


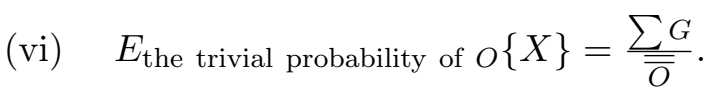

(36) Let $X$ be a real-valued random variable of $S$. Suppose $0<r$ and $X$ is non-negative and $X$ is integrable on $P$. Then $P(\{t \in O: r \leq X(t)\}) \leq$ $\frac{E_{P}\{X\}}{r}$.

\section{REFERENCES}

[1] Grzegorz Bancerek. Cardinal numbers. Formalized Mathematics, 1(2):377-382, 1990.

[2] Grzegorz Bancerek. The fundamental properties of natural numbers. Formalized Mathematics, 1(1):41-46, 1990.

[3] Grzegorz Bancerek. The ordinal numbers. Formalized Mathematics, 1(1):91-96, 1990.

[4] Grzegorz Bancerek and Krzysztof Hryniewiecki. Segments of natural numbers and finite sequences. Formalized Mathematics, 1(1):107-114, 1990.

[5] Józef Białas. Infimum and supremum of the set of real numbers. Measure theory. Formalized Mathematics, 2(1):163-171, 1991.

[6] Józef Białas. Series of positive real numbers. Measure theory. Formalized Mathematics, 2(1):173-183, 1991.

[7] Józef Białas. The $\sigma$-additive measure theory. Formalized Mathematics, 2(2):263-270, 1991.

[8] Józef Białas. Some properties of the intervals. Formalized Mathematics, 5(1):21-26, 1996.

[9] Czesław Byliński. Functions and their basic properties. Formalized Mathematics, 1(1):5565, 1990.

[10] Czesław Byliński. Functions from a set to a set. Formalized Mathematics, 1(1):153-164, 1990.

[11] Czesław Byliński. Partial functions. Formalized Mathematics, 1(2):357-367, 1990.

[12] Czesław Byliński. Some basic properties of sets. Formalized Mathematics, 1(1):47-53, 1990.

[13] Czesław Byliński. The sum and product of finite sequences of real numbers. Formalized Mathematics, 1(4):661-668, 1990.

[14] Agata Darmochwał. Finite sets. Formalized Mathematics, 1(1):165-167, 1990.

[15] Noboru Endou and Yasunari Shidama. Integral of measurable function. Formalized Mathematics, 14(2):53-70, 2006, doi:10.2478/v10037-006-0008-x.

[16] Noboru Endou, Katsumi Wasaki, and Yasunari Shidama. Basic properties of extended real numbers. Formalized Mathematics, 9(3):491-494, 2001.

[17] Noboru Endou, Katsumi Wasaki, and Yasunari Shidama. Definitions and basic properties of measurable functions. Formalized Mathematics, 9(3):495-500, 2001.

[18] Krzysztof Hryniewiecki. Basic properties of real numbers. Formalized Mathematics, 1(1):35-40, 1990.

[19] Grigory E. Ivanov. Definition of convex function and Jensen's inequality. Formalized Mathematics, 11(4):349-354, 2003.

[20] Jarosław Kotowicz. Real sequences and basic operations on them. Formalized Mathematics, 1(2):269-272, 1990.

[21] Jarosław Kotowicz and Yuji Sakai. Properties of partial functions from a domain to the set of real numbers. Formalized Mathematics, 3(2):279-288, 1992.

[22] Keiko Narita, Noboru Endou, and Yasunari Shidama. Integral of complex-valued measurable function. Formalized Mathematics, 16(4):319-324, 2008, doi:10.2478/v10037-0080039-6.

[23] Andrzej Nędzusiak. Probability. Formalized Mathematics, 1(4):745-749, 1990.

[24] Andrzej Nędzusiak. $\sigma$-fields and probability. Formalized Mathematics, 1(2):401-407, 1990.

[25] Jan Popiołek. Introduction to probability. Formalized Mathematics, 1(4):755-760, 1990.

[26] Yasunari Shidama and Noboru Endou. Integral of real-valued measurable function. Formalized Mathematics, 14(4):143-152, 2006, doi:10.2478/v10037-006-0018-8.

[27] Andrzej Trybulec. On the sets inhabited by numbers. Formalized Mathematics, 11(4):341$347,2003$.

[28] Zinaida Trybulec. Properties of subsets. Formalized Mathematics, 1(1):67-71, 1990.

[29] Edmund Woronowicz. Relations defined on sets. Formalized Mathematics, 1(1):181-186, 1990. 
[30] Bo Zhang, Hiroshi Yamazaki, and Yatsuka Nakamura. The relevance of measure and probability, and definition of completeness of probability. Formalized Mathematics, 14(4):225-229, 2006, doi:10.2478/v10037-006-0026-8.

Received March 17, 2009 